\title{
Oxide and Carbide Formation at Titanium/Organic Monolayer Interfaces
}

\section{Supporting Information}

Jason J. Blackstock, ${ }^{\dagger}$ Carrie L. Donley, ${ }^{\dagger \S}$ William F. Stickle, ${ }^{\ddagger}$

Douglas A.A. Ohlberg, ${ }^{\dagger}$ J. Joshua Yang, ${ }^{\dagger}$ Duncan R. Stewart ${ }^{\dagger{ }^{*}}$ and R. Stanley Williams ${ }^{\dagger}$

${ }^{\dagger}$ Information and Quantum Science Laboratory, Hewlett-Packard Labs, 1501 Page Mill Rd, Palo Alto, CA, USA

‡ Analytical and Development Labs, Hewlett-Packard Company, Corvallis, OR, USA

*duncan.stewart@hp.com

${ }^{\S}$ Current Address: University of North Carolina, Institute for Advanced Materials, 243 Chapman Hall, Chapel Hill, NC 27599 


\section{a Ti(2p) angle-resolved XPS \\ 'top' of SiO2 sample}

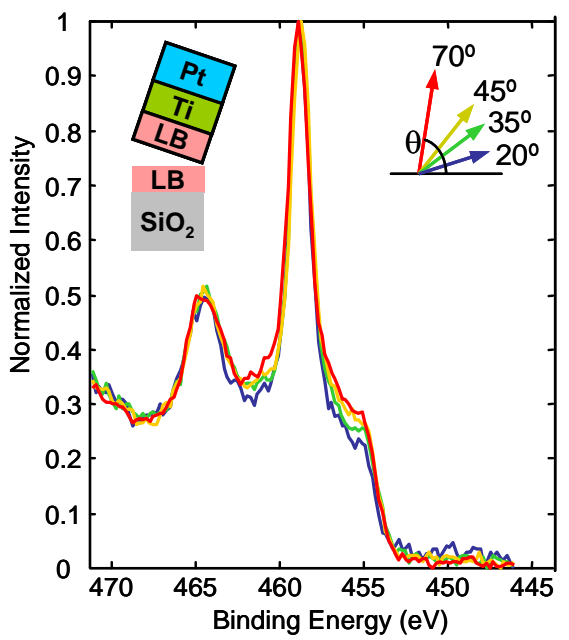

\section{d Ti(2p) angle-resolved XPS 'top' of PtOx sample}

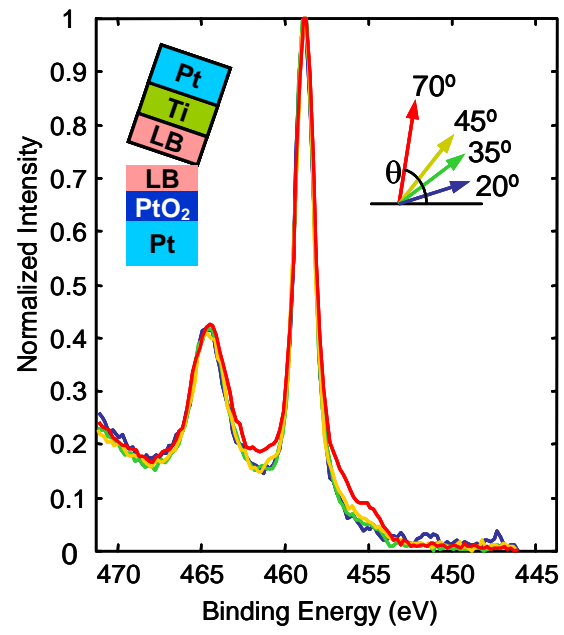

\section{b C(1s) angle-resolved XPS 'top' of SiO2 sample}

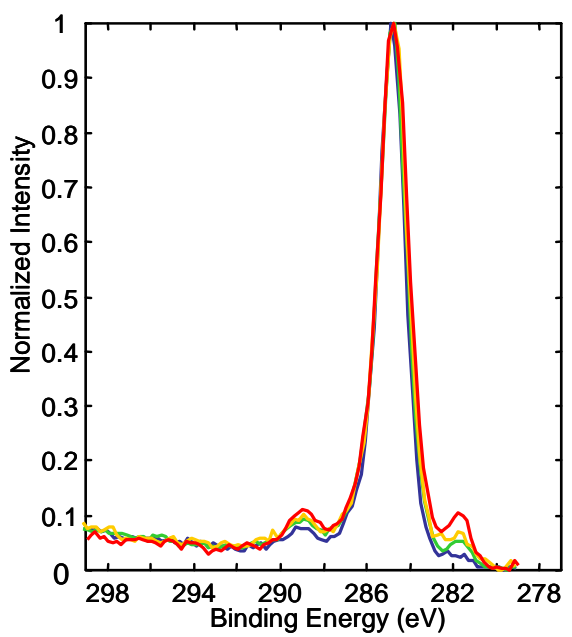

e $\quad \mathbf{C ( 1 s )}$ angle-resolved XPS
'top' of PtOx sample

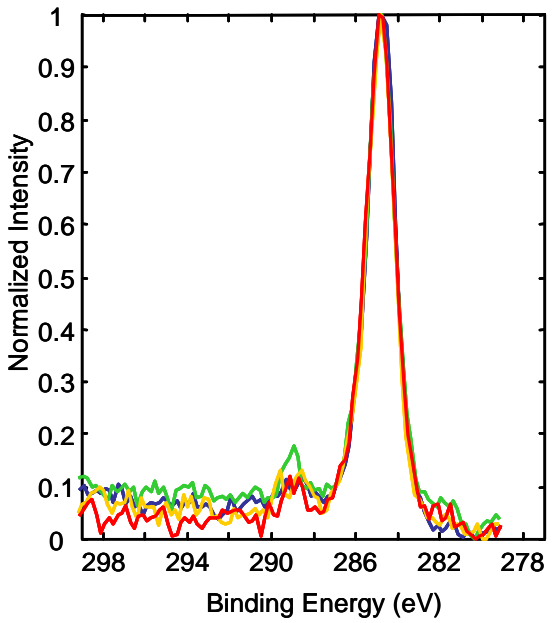

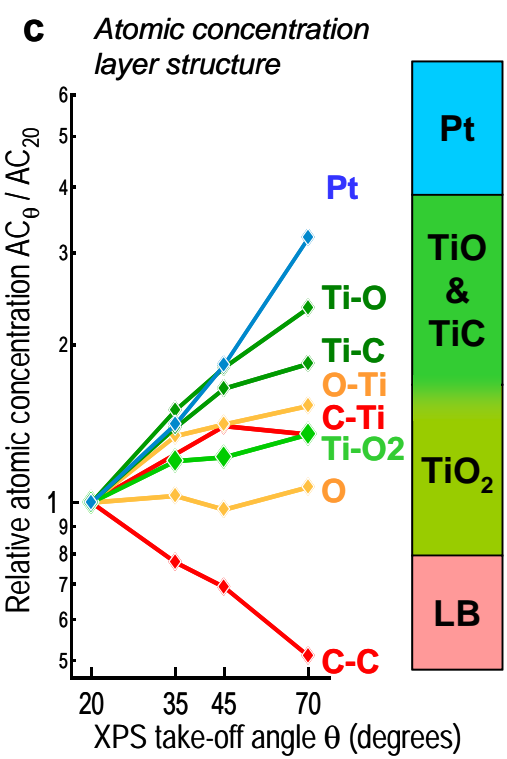

f Atomic concentration layer structure

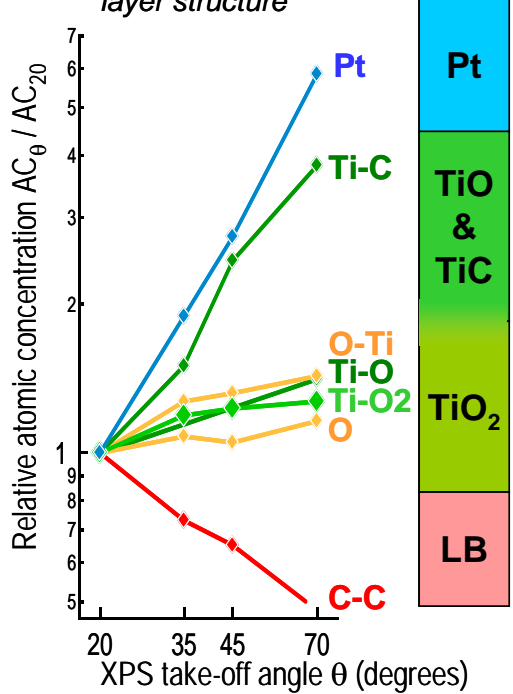

Figure S1. Complementary data to Figure 3 in the main paper, for the SiO2 and PtOx substrates. (a) Ti(2p) angled resolved AR-XPS spectra from the delaminated 'top' surface of the SiO2 substrate sample shows the TiC and TiO peaks increase with XPS take-off angle, indicating they are buried below the TiO2. (b) C(1s) AR-XPS shows the same trend for the TiC peak. (c) Quantification of atomic concentrations as a function of angle enable a simplified layer model to be constructed. The LB film is identified with the surface $\mathrm{C}-\mathrm{C}$ carbon signature; the Ti oxide and Ti carbide are visible in the $\mathrm{Ti}(2 \mathrm{p})$ spectra (Ti-O, Ti-C, TiO2), the carbon $\mathrm{C}(1 \mathrm{~s})$ spectra $(\mathrm{C}-\mathrm{Ti})$ and the oxygen $\mathrm{O}(1 \mathrm{~s})$ spectra $(\mathrm{O}, \mathrm{O}-\mathrm{Ti})$. The $\mathrm{Pt}(4 \mathrm{f})$ signal is from the $\mathrm{Pt}$ capping layer. In particular, this atomic concentration dependence confirms that the TiOx and $\mathrm{TiC}$ reside above the $\mathrm{TiO} 2$ in the fabricated structure. $(d, e, f)$ Corresponding data for the PtOx substrate. 


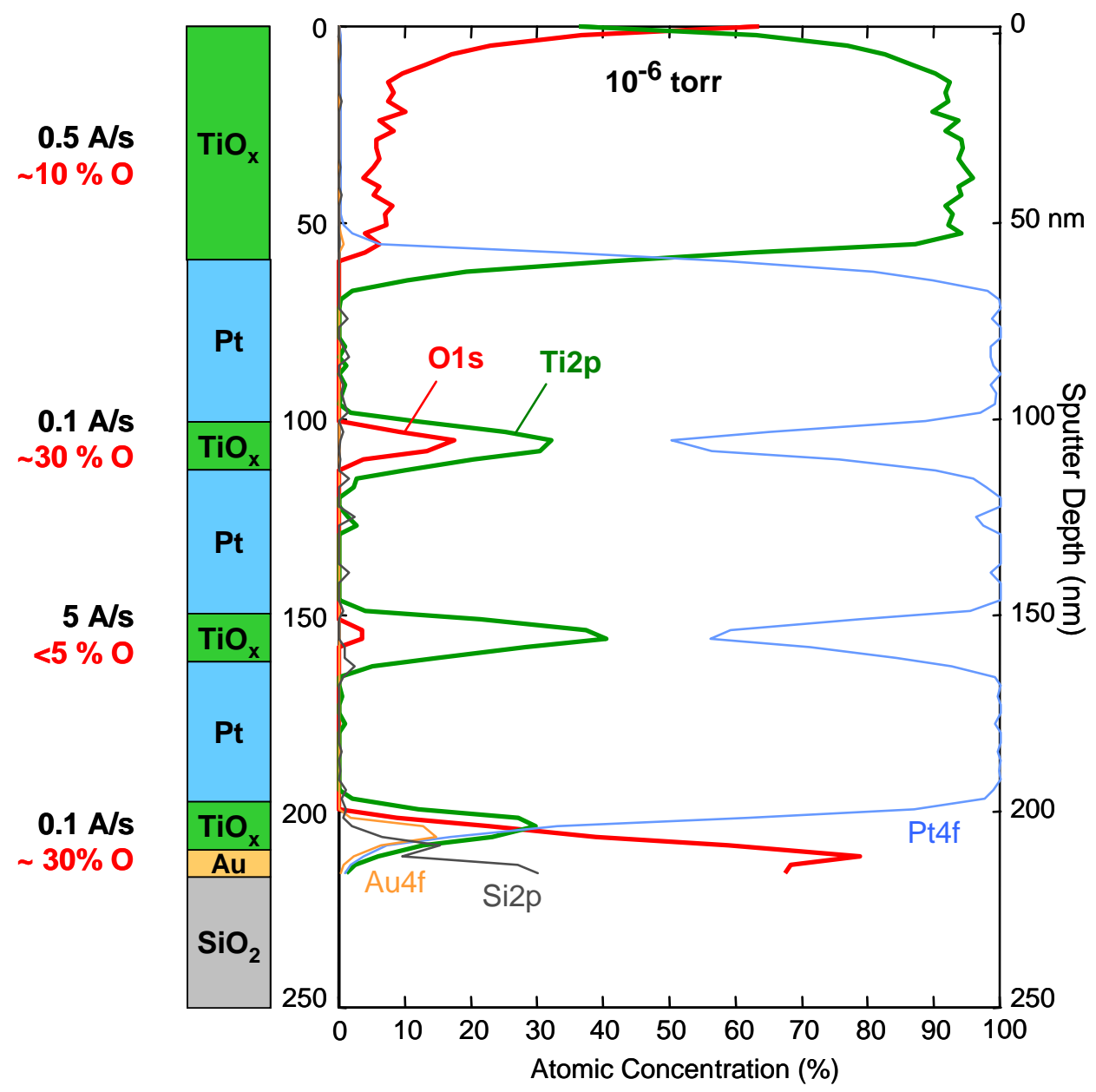

Figure S2. TiOx formation as a function of deposition rate at a chamber pressure of 10-6 torr. Successive layers of Ti were deposited at different deposition rates as illustrated without breaking vacuum. X-ray photoemission spectra were aquired during subsequent Zalar rotation ion milling of the sample using $1 \mathrm{keV}$ Ar ions. Atomic concentrations were calculated from the XPS spectra via conventional sensitivity correction. The Ti (green) and $\mathrm{O}$ (red) atomic concentrations can be used to reach approximate O/Ti ratios for the different deposition rate $\mathrm{Ti}$ thin films, written at left. Slow depositions at $0.1 \mathrm{~A} / \mathrm{s}$ are typical for our Ti-onto-organic system, but yield a TiOx material where $x$ can be as high as 0.5 , i.e. $~ 30 \%$ oxygen content in the deposited film. Ti deposited at high rates of $5 \mathrm{~A} / \mathrm{s}$ show very little oxygen incorporation, $<5 \%$ total $\mathrm{O}$ content. 